\title{
Sleep increases explicit solutions and reduces intuitive judgments of semantic coherence
}

\author{
Thea Zander, ${ }^{1}$ Kirsten G. Volz, ${ }^{2}$ Jan Born, ${ }^{3,4}$ and Susanne Diekelmann ${ }^{3}$ \\ ${ }^{1}$ Department of Psychology, University of Basel, Basel 4055, Switzerland; ${ }^{2}$ Clinic of Psychiatry, Psychotherapy, and Psychosomatics, \\ Reutlingen 72764, Germany; Institute of Medical Psychology and Behavioral Neurobiology, University of Tübingen 72076, Germany; \\ ${ }^{4}$ Werner Reichardt Centre for Integrative Neuroscience, University of Tübingen 72076, Tübingen, Germany
}

\begin{abstract}
Sleep fosters the generation of explicit knowledge. Whether sleep also benefits implicit intuitive decisions about underlying patterns is unclear. We examined sleep's role in explicit and intuitive semantic coherence judgments. Participants encoded sets of three words and after a sleep or wake period were required to judge the potential convergence of these words on a common fourth associate. Compared with wakefulness, sleep increased the number of explicitly named common associates and decreased the number of intuitive judgments. This suggests that sleep enhances the extraction of explicit knowledge at the expense of the ability to make intuitive decisions about semantic coherence.
\end{abstract}

[Supplemental material is available for this article.]

In everyday life we sometimes make decisions based on a "gut feeling" without knowing the exact reasons for why we decided in this way. Intuitive processing has been conceptualized as the preliminary detection of meaning in the environment by recognizing "some pattern, structure, or organization [that] exists prior to its detection" (Bowers et al. 1997). Intuitive processes are assumed to draw on tacit knowledge stored in semantic memory networks. This tacit knowledge may be momentarily inaccessible to conscious awareness, but can be activated by environmental cues (Yaniv and Meyer 1987; Bowers et al. 1990).

In a classical task measuring intuition, participants are asked to make semantic coherence judgments on three presented words, like SALT DEEP FOAM (e.g., Bolte and Goschke 2005; Topolinski and Strack 2009). Semantic coherence is defined by the existence of a fourth word that the word triad's constituents (remotely) have in common, the common associate (OCEAN in this example). Studies have shown that participants perform above chance level in correctly judging coherent triads as coherent even if they are not able to name the common associate. This process of generating the feeling of semantic associativity has been conceptualized in the framework of automatic spread of activation. According to this, the encounter of the triad automatically triggers the activation of related concepts stored in semantic memory resulting in a coherence impression. This first implicit coherence impression can become consciously accessible if enough activation has accumulated to cross a threshold of awareness resulting in the ability to explicitly name the common associate (Bowers et al. 1990). Even though intuitive decision making has been behaviorally described in several studies (e.g., Bolte and Goschke 2005; Topolinski and Strack 2009), the physiological mechanisms underlying this phenomenon are largely unknown (Volz and Zander 2014).

Sleep is well-known to facilitate the consolidation of newly encoded material by strengthening and integrating new memory representations into the long-term store (e.g., Diekelmann and Born 2010; Stickgold and Walker 2013). For example, sleep facilitates the generalization and abstraction of schema-like information from single encoded elements (Lewis and Durrant 2011).

\section{Corresponding author: thea.zander@unibas.ch}

Article is online at http://www.learnmem.org/cgi/doi/10.1101//m.044511.116.
Sleep also supports the conversion of implicitly learned regularities into explicit knowledge about those regularities, allowing for "insight" into underlying patterns (Fischer et al. 2006; Yordanova et al. 2008; Drosopoulos et al. 2011; Verleger et al. 2013). Along these lines, Monaghan et al. (2015) observed that sleep improves analogical transfer in problem solving by abstracting and structurally generalizing rules and/or task solutions across different problems. Such processes are assumed to rely on the covert reactivation ("replay") of learning-related neuronal activity during sleep (Cousins et al. 2014; Diekelmann et al. 2016).

Sleep also enhances associative memory processes (Stickgold et al., 1999). Two recent sleep studies applied the remote associate task (RAT), in which participants are asked to find a semantic concept that forms a link between three presented words (for instance HEART SIXTEEN COOKIE, solution: SWEET). The RAT is similar to the semantic coherence task described above but tests for explicit solutions only, while not assessing intuitive responses. Cai et al. (2009) found that sleep facilitates the ability to explicitly name solution words in the RAT, yet only when solution words were primed before sleep. Sio et al. (2013), using similar RAT stimuli, showed that sleep can increase the generation of explicit solutions even without priming, but this effect was restricted to difficult RAT items. Although these studies provide a first hint that under certain conditions sleep increases the generation of explicit RAT solutions, the effect of sleep on intuitive judgments remains unknown.

Here, we asked whether sleep affects intuitive and explicit semantic coherence judgments. We hypothesized that sleep increases the number of explicit responses as well as the number of intuitive judgments. This was based on the automatic spread of activation account: We assumed that concepts, comprising core and remote associations, with a relatively strong activation before sleep would be boosted during sleep to cross the threshold of awareness, resulting in more explicit solutions. Concurrently, weakly activated concepts should be boosted during sleep as well, such that after

(C) 2017 Zander et al. This article is distributed exclusively by Cold Spring Harbor Laboratory Press for the first 12 months after the full-issue publication date (see http://learnmem.cshlp.org/site/misc/terms.xhtml). After 12 months, it is available under a Creative Commons License (AttributionNonCommercial 4.0 International), as described at http://creativecommons. org/licenses/by-nc/4.0/. 
sleep they are activated enough to energize intuitive decisions, leading to a higher number of intuitive coherence judgments.

Altogether 31 healthy, German native-speaking, righthanded students participated in the experiment. They were randomly allocated to one of two groups, a sleep group $(n=15,8 \mathrm{fe}-$ male) and a wake group ( $n=16,10$ female). One sleep participant was excluded because he reported regular sleep disturbances. Mean age did not differ between groups (sleep group: $24.07 \pm$ 3.32 (SD); wake group: $24.13 \pm 4.12 ; t=-0.043, \mathrm{df}=29, p=0.97$ ). Females did not use any hormonal contraceptives and the experiments were scheduled in the mid-luteal phase of their menstrual cycle, in order to control for previously reported effects of menstrual cycle phase and hormonal contraceptives on sleep-dependent memory consolidation (Genzel et al. 2012, 2014). Further exclusion criteria were: medication, nightshifts for at least 8 wks before the experiment, sleep disturbances or irregular sleep-wake cycle, neurological or sleep-related diseases, being involved in the preparation of an imminent exam, and caffeine/alcohol intake on the experimental day. Participants were instructed not to take naps during the experimental day. All participants gave written informed consent and the study was approved by the local ethics committee of the Medical Faculty of the University of Tübingen.

The experiment consisted of two sessions, initial encoding in the first session and testing in the second session. During the retention interval between encoding and testing, participants either slept $8 \mathrm{~h}$ during the night (sleep group) or stayed awake during the day (wake group) (Fig. 1A). Participants in the sleep group came to the laboratory in the evening ( 22:00) and were prepared for polysomnographic recordings. To control for general alertness, they performed a vigilance task assessing reaction times and error rates (Roach et al. 2006) and rated their subjective sleepiness on the Stanford Sleepiness Scale (Hoddes et al. 1973). Subsequently, encoding of the word triads took place, and thereafter participants

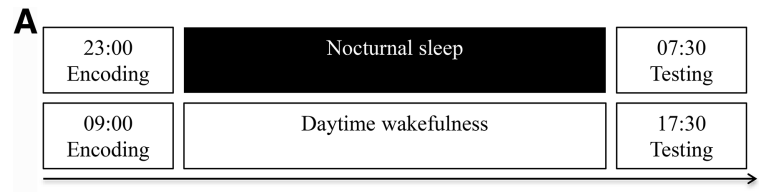

B

\begin{tabular}{l}
$\begin{array}{l}\text { Shallow Encoding: } \\
\text { Count the number of vowels } \\
\text { in the three words. }\end{array}$ \\
\hline \begin{tabular}{ll} 
SALT & Number \\
DEEP & of vowels? \\
FOAM & \\
\hline
\end{tabular} \\
\hline
\end{tabular}

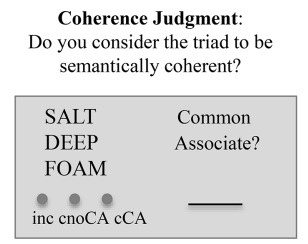

Coherence Judgment:
Do you consider the triad to be

were allowed to sleep for $8 \mathrm{~h}$ in the laboratory, with polysomnographic data continuously being recorded. Standard polysomnography included electroencephalography (EEG, at positions C3 and C4), electrooculography (EOG), and electromyography (EMG). Sleep stages were determined offline according to the standard criteria by Rechtschaffen and Kales (1968). Participants showed normal sleep patterns with a total sleep time of $443.67 \pm 9.34 \mathrm{~min}$ (see Supplemental Table S1 for detailed sleep data). During testing in the morning ( 07:30), participants performed the semantic coherence judgment task first and were then tested on their explicit memory for the encoded word triads in a cued recall and a recognition task. Participants then performed the vigilance task and rated their subjective sleepiness again (see Supplemental Table S2 for all control data; none of the control data revealed any differences between groups). The wake group started encoding in the morning ( 09:00), following the same procedure as the sleep group. After the encoding session, participants left the laboratory and went about their daily activities. Testing took place in the early evening ( 17:30), again following the same procedure as the sleep group.

For the semantic coherence judgment task, participants shallowly encoded 200 word triads (100 coherent, 100 incoherent; Fig. 1B). Encoding was embedded in a vowel counting task. The three words of each triad were presented for either $2 \mathrm{sec}$ (short encoding) or $4 \mathrm{sec}$ (long encoding) and subjects were instructed to count the number of vowels in the three words and give their response within $3 \mathrm{sec}$. At testing after the sleep/wake interval, half of the previously encoded word triads (50 coherent and 50 incoherent) were randomly presented for $4 \mathrm{sec}$ and subjects had to indicate whether the triad was semantically coherent or incoherent. Irrespective of their response, participants were asked to type in a common associate right after their coherence judgment. Common associates were counted as correct if the exact word or a plausible synonym was produced. For explicit coherence judgments, the percentage of correctly produced common associates was calculated, irrespective of prior coherence judgments. To assess intuitive coherence judgments, we computed the intuition index defined as the difference between hits (coherent triads correctly classified as coherent, but without a correct common associate) and false alarms (incoherent triads falsely classified as co-

C

Cued Recall Recognition

\begin{tabular}{|lr|}
\hline SALT & Words? \\
& \\
& \\
\hline
\end{tabular}

Figure 1. Schematic overview of the experimental procedure and the tasks. (A) The sleep group encoded the word triads in the evening and was tested in the next morning after $8 \mathrm{~h}$ of sleep. For the wake group, encoding and testing took place in the morning and evening, respectively. (B) For the semantic coherence judgment task, shallow encoding of 200 word triads ( 100 coherent, 100 incoherent) was embedded in a vowel counting task. Word triads were presented for either $2 \mathrm{sec}$ (short encoding) or $4 \mathrm{sec}$ (long encoding) and participants were asked to type in the overall number of vowels for each triad. At testing, half of the encoded triads (50 coherent and 50 incoherent) were presented again in a coherence judgment task and participants had to indicate whether the triad was semantically coherent or incoherent. They were instructed that coherent triads have a fourth word in common that characterizes the semantic link between its constituents, that is, a common associate. There were three response options: (inc) the triad is incoherent, (cnoCA) the triad is coherent, but a common associate cannot be retrieved immediately, (cCA) the triad is coherent and a common associate can be retrieved immediately. Irrespective of their response, participants were asked to type in a common associate right after their coherence judgment. (C) After the semantic coherence judgment, participants' explicit memory for the encoded word triads was tested. In a cued recall test, the other half of the previously encoded word triads (50 coherent and 50 incoherent) was tested. The first word of each triad was presented and participants were asked to type in the second, third, or both corresponding words. The subsequent recognition test consisted of all of the 200 encoded word triads intermixed with 200 new word triads, for which participants had to indicate whether each triad was old (i.e., presented before) or new. herent) (cf. Bolte et al. 2003). Intuitive coherence judgments were only computed for triads for which participants did not come up with a correct common associate, therefore explicit coherence judgments and the intuition index consist of different trial types (note that both measures were not correlated with each other, in the overall sample, $r=-0.13, p=0.49$, as well as in the sleep group, $r=0.06, p=$ 0.84 , and in the wake group, $r=0.25, p=$ 0.36 , separately).

The overall response pattern of the coherence judgments is illustrated in Table 1. Analysis of explicit coherence judgments showed that sleep participants produced significantly more common associates than wake participants (sleep: $50.74 \pm 3.97 \%$, wake: $40.53 \pm 2.38 \%$; main effect "sleep/wake": $F_{(1,28)}=5.15$, $p=0.031)$, suggesting that the likelihood of becoming aware of explicit solutions was higher after sleep than wakefulness (Fig. 2A). This sleep-wake difference was 
Table 1. Response pattern in the semantic coherence judgment task

\begin{tabular}{|c|c|c|c|c|c|}
\hline & & \multicolumn{2}{|c|}{ Sleep group } & \multicolumn{2}{|c|}{ Wake group } \\
\hline & & Judgment & Common associate & Judgment & Common associate \\
\hline Coherent & Incoherent $^{\mathrm{a}}$ & $27.64 \pm 4.87$ & $9.83 \pm 2.01$ & $21.35 \pm 4.06$ & $8.10 \pm 1.57$ \\
\hline \multirow{2}{*}{ Short encoding } & Coherent no common associate $^{\mathrm{b}}$ & $17.18 \pm 4.47$ & $11.55 \pm 3.92$ & $21.33 \pm 5.95$ & $10.68 \pm 4.12$ \\
\hline & Coherent common associate ${ }^{b}$ & $54.90 \pm 7.10$ & $50.86 \pm 6.67$ & $57.05 \pm 6.85$ & $41.94 \pm 5.51$ \\
\hline Coherent & Incoherent ${ }^{\mathrm{a}}{ }^{2}$ & $66.40 \pm 5.36$ & $13.53 \pm 3.40$ & $53.26 \pm 5.74$ & $8.60 \pm 1.86$ \\
\hline \multirow{2}{*}{ Long encoding } & Coherent no common associate ${ }^{b}$ & $13.33 \pm 4.16$ & $4.85 \pm 2.59$ & $16.69 \pm 3.05$ & $3.03 \pm 0.85$ \\
\hline & Coherent common associate $^{\mathrm{b}}$ & $20.00 \pm 4.71$ & $11.17 \pm 3.00$ & $29.53 \pm 5.40$ & $8.83 \pm 1.60$ \\
\hline Incoherent & Incoherent $^{\mathrm{c}}$ & $81.06 \pm 4.44$ & & $71.69 \pm 4.47$ & \\
\hline \multirow[t]{2}{*}{ Short encoding } & Coherent no common associate ${ }^{d}$ & $9.86 \pm 2.78$ & & $11.21 \pm 2.20$ & \\
\hline & Coherent common associate ${ }^{d}$ & $9.06 \pm 3.23$ & & $17.09 \pm 3.39$ & \\
\hline Incoherent & Incoherent $^{\mathrm{c}}$ & $76.47 \pm 4.86$ & & $66.82 \pm 4.91$ & \\
\hline \multirow{2}{*}{ Long encoding } & Coherent no common associate ${ }^{d}$ & $10.16 \pm 3.35$ & & $14.83 \pm 3.64$ & \\
\hline & Coherent common associate $\mathrm{d}^{\mathrm{d}}$ & $13.08 \pm 3.13$ & & $18.09 \pm 4.32$ & \\
\hline
\end{tabular}

For both groups (sleep/wake), the judgment as the percentages of the three possible response categories: incoherent, coherent but no common associate known (coherent no common associate), and coherent and common associate immediately known (coherent common associate) are indicated, separately for coherent triads and incoherent triads as well as for triads that had been encoded for $2 \mathrm{sec}$ (short encoding) or $4 \mathrm{sec}$ (long encoding). Additionally, the percentages of correctly produced common associates are indicated for each response category for coherent triads (note that participants were asked to produce a possible common associate irrespective of their initial judgment, thus, some participants produced correct common associates despite initially judging the triad as incoherent or as coherent without immediately knowing the common associate). There was no correct common associate for incoherent triads. The number of incorrectly produced common associates was $\sim 2.6 \%$ for incoherent triads and $\sim 8.5 \%$ for coherent triads, across all groups and conditions. Means \pm SEM are shown. ${ }^{\mathrm{a}}$ Misses, number of coherent triads that were falsely classified as incoherent.

${ }^{\mathrm{b}} \mathrm{Hits}$, number of coherent triads that were correctly classified as coherent (regardless of the common associate).

${ }^{c}$ Correct rejection, number of incoherent triads that were correctly categorized as incoherent.

${ }^{\mathrm{d}}$ False alarms, number of incoherent triads that were falsely judged as coherent.

not affected by the duration of encoding (interaction effect "sleep/ wake" $\times$ "short/long": $\left.F_{(1,28)}=0.19, p=0.66\right)$. However, triads that had been encoded for 2 sec before the sleep/wake interval had a generally higher likelihood of being explicitly solved than triads that had been encoded for 4 sec (short: $65.96 \pm 3.16 \%$, long: $24.64 \pm 2.43 \%$; main effect "short/long": $F_{(1,28)}=189.28, p<$ $0.001)$.

In contrast to explicit judgments, sleep participants showed significantly fewer intuitive judgments than wake participants (sleep: $8.77 \pm 2.41 \%$, wake: $21.16 \pm 2.16 \%$; main effect "sleep/ wake": $\left.F_{(1,28)}=14.71, p<0.001\right)$, suggesting that sleep compared with wakefulness distinctly reduced the ability to make intuitive decisions (Fig. 2B). Importantly, both intuition indices, for the sleep and wake groups, were significantly higher than chance level (chance level $=0$; sleep: $t_{(13)}=3.63, \quad p<0.005$; wake: $\left.t_{(15)}=9.78, p<0.001\right)$. Since the intuition index is calculated as the difference between hits and false alarms, we analyzed these measures separately to examine whether the lower intuition index in the sleep group resulted from lower hits or from higher false alarms. Sleep participants showed significantly fewer hits compared with wake participants (29.41 $\pm 5.41 \%$ versus $51.77 \pm 5.16 \%$; $t_{(28)}=$ $-2.89, p=0.006)$, whereas false alarms did not differ significantly (20.64 \pm $4.61 \%$ versus $30.60 \pm 4.51 \% ; \quad t_{(28)}=$ $-1.53, p=0.14)$. Like for explicit responses, the difference between sleep and wakefulness in the intuition index was independent of the encoding duration (interaction effect "sleep/wake" × "short/ long": $\left.F_{(1,28)}=0.41, p=0.52\right)$, but a significantly higher intuition index was observed for triads that were encoded for 2 sec than for triads that were encoded for $4 \mathrm{sec}$ before the sleep or wake interval (short: $34.12 \pm 4.64 \%$, long: $6.25 \pm 2.58 \%$; main effect "short/long": $\left.F_{(1,28)}=27.89, p<0.001\right)$.

To test for explicit memory of the encoded word triads, a cued recall test and a recognition test were performed after the semantic coherence task (Fig. 1C). For cued recall, the first word of half of the encoded word triads ( 50 coherent and 50 incoherent) was presented and subjects were asked to name the second and/or third word of the triad. Overall cued recall performance was very low and there was no difference between the sleep and wake groups (number of recalled words, sleep: $1.90 \pm 0.42$, wake: $2.10 \pm 0.48$; all $p>0.17$ ).
A

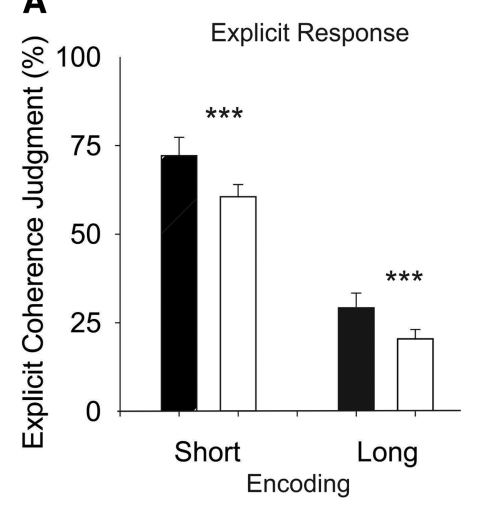

B

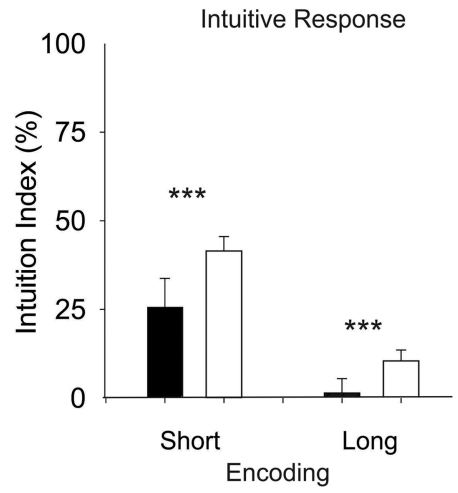

$$
\begin{array}{r}
\longleftarrow \text { Sleep Group } \\
\text { Wake Group }
\end{array}
$$

Figure 2. Explicit responses $(A)$ and intuitive responses $(B)$ for the sleep and wake groups. The sleep group produced more explicit responses and less intuitive responses, independent of encoding duration. Explicit responses refer to the percentage of correctly named common associates for coherent triads. Intuitive responses were assessed with the intuition index for coherent triads for which participants were not able to name a common associate. The intuition index is calculated as hit rate ("coherent" judgment on coherent triads) minus false alarm rate ("coherent" judgment on incoherent triads). (Short encoding) triads that were encoded for $2 \mathrm{sec}$; (long encoding) triads that were encoded for 4 sec. Means \pm SEM are shown. ${ }^{* * *} p<0.001$. 
Cued recall was also independent of the type of triad (coherent/incoherent) as well as of encoding duration (short/long) (all $p>0.10$ ). In the recognition test, all encoded word triads (100 coherent and 100 incoherent) were presented again together with new triads and subjects were asked to indicate for each triad whether it was old (i.e., encoded before) or new. Hits were defined as the percentage of old triads correctly recognized as old and false alarms were defined as the percentage of new triads falsely judged as old. The number of hits and false alarms was not different between the sleep group (hits: $45.20 \pm 4.39 \%$; false alarms: $14.57 \pm 2.66 \%$ ) and the wake group (hits: $51.60 \pm 2.75 \%$; false alarms: $14.65 \pm 1.94 \%$; both $p>0.22$ ) or between encoding duration (short/long) (all $p>$ 0.51 ). Across both encoding periods (short/long) and both groups (sleep/wake), participants recognized coherent triads (sleep: 47.06 $\pm 4.19 \%$, wake: $53.63 \pm 2.20 \%$ ) better than incoherent triads (sleep: $43.33 \pm 4.69 \%$, wake: $49.84 \pm 3.53 \%$ ), main effect type of triad: $F_{(1,28)}=7.28, p=0.012$.

These findings show that, contrary to our hypothesis, sleep affected explicit and intuitive coherence judgments differently. When compared with the wakefulness retention interval, sleep resulted in a higher number of explicitly named common associates. However, sleep did not benefit but even diminished the ability to make intuitive judgments about semantic coherence. Interestingly, independent of the effect of sleep, both explicit and intuitive judgments were higher if the word triads had been encoded for $2 \mathrm{sec}$ when compared with $4 \mathrm{sec}$.

Our data are consistent with previous research, showing that sleep selectively benefits memory representations that are in any way relevant or important to the individual: Sleep preferentially consolidates associative memories expected to be relevant for a retrieval test after sleep (Wilhelm et al. 2011). Similarly, sleep favors the consolidation of procedural memories for which participants anticipated to be rewarded at testing after sleep (Fischer and Born 2009). Memories that are emotionally salient are likewise preferentially enhanced during sleep, at the expense of neutral information, a phenomenon known as "emotional trade-off" (Payne et al. 2008; Payne and Kensinger 2010). Based on this evidence, we speculate that in our study sleep-dependent processing favored associations that appeared to be more relevant or important and suppressed irrelevant associations, with relevance possibly being signaled by the relative strength of the encoded associations. It has been suggested that sleep particularly favors associations with a medium encoding strength (Stickgold 2009; Wilhelm et al. 2012), while associations that are too weak or too strong do not benefit from sleep (Drosopoulos et al. 2007; Tucker and Fishbein 2008). Considering that in the present study, word triads were encoded shallowly and incidentally, the overall encoding strength was presumably moderate, such that relatively stronger associations fell within the medium strength range, while relatively weaker associations may have been too weak to benefit from sleep. Accordingly, sleep might have preferentially facilitated semantic activations of concepts that had a sufficiently strong activation before sleep, resulting in an accumulation of activation in the semantic memory network. This accumulation of activation during sleep may have helped the respective semantic concepts to cross the threshold of awareness, leading to an increase in the number of explicitly named common associates after sleep.

Semantic associations that were only very weakly activated before sleep, on the other hand, might have been weakened further and respective increases in connective strength from encoding might even have become nullified during sleep. It has been suggested that sleep has an important function not only in the strengthening of relevant information but also in the forgetting of irrelevant information (Crick and Mitchison 1983). The synaptic homeostasis hypothesis proposes that a central function of sleep is the restoration of synaptic homeostasis (Tononi and
Cirelli 2006, 2014). While wakefulness is characterized by an increase in synaptic strength through constant learning, sleep renormalizes the overall synaptic strength in a process of "downselection" (Tononi and Cirelli 2014). During this process, all synaptic connections become downscaled, while relatively strong connections might be downscaled to a lesser extent or might even be entirely protected from downscaling and very weak connections might be erased completely (Hashmi et al. 2013; Nere et al. 2013). Importantly, the basis for intuitive decisions is that weak activations of semantic concepts produce a feeling of coherence strong enough to decide upon intuitively even though no explicit reasons can be given. If sleep reduces or even eliminates these weak activations, there is not enough activation left to nurse the hunch necessary for intuition. Thus, sleep might help us extract explicit knowledge by strengthening sufficiently activated associations, but concurrently erases the basis for intuitive judgments by eliminating weakly activated associations.

Apart from the effect of sleep on explicit and intuitive judgments, we also observed a striking but unexpected effect of the encoding duration. With a longer encoding duration, participants produced fewer common associates as well as fewer intuitive coherence judgments compared with short encoding. In line with previous evidence, it could be speculated that the longer encoding duration led to deeper conscious processing of the word triads, which may have disrupted or interfered with early automatic semantic activation of the common associates (Topolinski and Strack 2008). This idea should be systematically tested in future studies.

A potential limitation of the present study relates to the comparison of nighttime sleep with daytime wakefulness and the corresponding circadian differences. Although we cannot fully exclude that circadian factors affected the present findings, we consider this unlikely as our control tasks (measuring general alertness, i.e., objective vigilance and subjective sleepiness) did not reveal any differences between groups. Moreover, a number of previous studies on sleep and memory, including one study on sleep and semantic processing, did not observe any circadian differences when directly controlling for such effects by introducing circadian control groups (e.g., Payne et al. 2008; Sio et al. 2013). As another potential limitation, it should be considered that the two measures assessing explicit solutions and intuitive judgments may not be entirely independent. Although both measures relied on different trial types and did not correlate, it is possible that the solved triads may have been somewhat easier, leaving the more difficult triads for the intuitive judgments. It is also possible that subjects judged certain triads as coherent intuitively at first, but then solved the triads within the time of giving the coherence judgment (yet this only rarely happened, in $\sim 8 \%$ of triads on average). As a final limitation, with the present experimental design, we cannot disentangle potential mechanisms underlying sleep's effect on semantic concepts. There are different possible scenarios how sleep may act on semantic processing; (1) sleep may strengthen the encoded words, increasing the likelihood that the common associate is generated spontaneously at testing, (2) sleep may directly strengthen the common associate that is generated already at encoding, (3) sleep may strengthen the associations between the encoded words and the concurrently activated common associate, or (4) sleep may directly generate the common associate via spreading (re-)activation of the encoded words, etc. These questions will be subject to future studies directly testing and manipulating potential influencing factors and mechanisms.

\section{Acknowledgments}

We thank Franziska Bröker for her help in data collection of the wake group as well as for her help in programming the vigilance 
task. The authors also thank Elena Roson Roman for help in programming the coherence task. This project was funded by the Werner Reichardt Centre for Integrative Neuroscience (CIN) at the University of Tübingen (an Excellence Cluster within the framework of the Excellence Initiative EXC 307) as well as by the Collaborative Research Centre "Plasticity and Sleep" (TR-SFB 654), funded by the Deutsche Forschungsgemeinschaft (DFG).

\section{References}

Bolte A, Goschke T. 2005. On the speed of intuition: Intuitive judgments of semantic coherence under different response deadlines. Mem Cognit 33: 1248-1255.

Bolte A, Goschke T, Kuhl J. 2003. Effects of positive and negative mood on implicit judgments of semantic coherence. Psychol Sci 14: 416-421.

Bowers KS, Regehr G, Balthazard C, Parker K. 1990. Intuition in the context of discovery. Cognitive Psychol 22: 72-110.

Bowers KS, Farvolden P, Mermigis L. 1997. Intuitive antecedents of insight. In The creative cognition approach (ed. Smith SM, Ward TB, Finke RA), pp. 27-51. MIT Press, Cambridge.

Cai DJ, Mednick SA, Harrison EM, Kanady JC, Mednick SC. 2009. REM, not incubation, improves creativity by priming associative networks. Proc Natl Acad Sci 106: 10130-10134.

Cousins JN, El-Deredy W, Parkes LM, Hennies N, Lewis PA. 2014. Cued memory reactivation during slow-wave sleep promotes explicit knowledge of a motor sequence. J Neurosci 34: 15870-15876.

Crick F, Mitchison G. 1983. The function of dream sleep. Nature 304: 111-114.

Diekelmann S, Born J. 2010. The memory function of sleep. Nat Rev Neurosci 11: $114-126$.

Diekelmann S, Born J, Rasch B. 2016. Increasing explicit sequence knowledge by odor cueing during sleep in men but not women. Front Behav Neurosci 10: 74

Drosopoulos S, Schulze C, Fischer S, Born J. 2007. Sleep's function in the spontaneous recovery and consolidation of memories. J Exp Psychol Gen 136: $169-183$.

Drosopoulos S, Harrer D, Born J. 2011. Sleep and awareness about presence of regularity speed the transition from implicit into explicit knowledge. Biol Psychol 86: 168-173.

Fischer S, Born J. 2009. Anticipated reward enhances offline learning during sleep. J Exp Psychol Learn Mem Cogn 35: 1586-1693.

Fischer S, Drosopoulos S, Tsen J, Born J. 2006. Implicit learning - explicit knowing: a role for sleep in memory system interaction. J Cogn Neurosci 18: $311-319$.

Genzel L, Kiefer T, Renner L, Ehrle R, Kruge M, Grözinger M, Steiger A, Dresler M. 2012. Sex and modulatory menstrual cycle effects on sleep related memory consolidation. Psychoneuroendocrinology 37: 987-998.

Genzel L, Bäurle A, Potyka A, Wehrle R, Adamczyk M, Friess E, Steiger A, Dresler M. 2014. Diminished nap effects on memory consolidation are seen under oral contraceptive use. Neuropsychobiology 70: 253-261.

Hashmi A, Nere A, Tononi G. 2013. Sleep dependent synaptic downselection (II): Single neuron level benefits for matching, selectivity, and specificity. Front Neurol 4: 148.

Hoddes E, Zarcone V, Smythe H, Phillips R, Dement W. 1973. Quantification of sleepiness: a new approach. Psychophysiology 10: 431-436.

Lewis PA, Durrant SJ. 2011. Overlapping memory replay during sleep builds cognitive schemata. Trends Cogn Sci 15: 343-351.
Monaghan P, Sio UN, Lau SW, Woo HK, Linkenauger SA, Ormerod T. 2015. Sleep promotes analogical transfer in problem solving. Cognition 143: 25-30.

Nere A, Hashimi A, Cirelli C, Tononi G. 2013. Sleep-dependent synaptic downselection (I): Modeling the benefits of sleep on memory consolidation and integration. Front Neurol 4: 143.

Payne JD, Kensinger EA. 2010. Sleep's role in the consolidation of emotional episodic memories. Curr Dir Psychol Sci 19: 290-295.

Payne JD, Stickgold R, Swanberg K, Kensinger EA. 2008. Sleep preferentially enhances memory for emotional components of scenes. Psychol Sci 19: 781-788.

Rechtschaffen A, Kales A. 1968. A manual of standardized terminology, techniques and scoring system for sleep stages of human subjects. US Department of Health, Education, and Welfare - National Institutes of Health, Bethesda, MD.

Roach GD, Dawson D, Lamond N. 2006. Can a shorter psychomotor vigilance task be used as a reasonable substitute for the ten-minute psychomotor vigilance task? Chronobiol Int 23: 1379-1387.

Sio UN, Monaghan P, Ormerod T. 2013. Sleep on it, but only if it is difficult: effects of sleep on problem solving. Mem Cogn 41: 159-166.

Stickgold R. 2009. How do I remember? Let me count the ways. Sleep Med Rev 13: $305-308$.

Stickgold R, Walker MP. 2013. Sleep-dependent memory triage: evolving generalization through selective processing. Nat Neurosci 16: 139-145.

Stickgold R, Scott L, Rittenhouse C, Hobson JA. 1999. Sleep-induced changes in associative memory. J Cogn Neurosci 11: 182-193.

Tononi G, Cirelli C. 2006. Sleep function and synaptic homeostasis. Sleep Med Rev 10: 49-62.

Tononi G, Cirelli C. 2014. Sleep and the price of plasticity: from synaptic and cellular homeostasis to memory consolidation and integration. Neuron 81: 12-34.

Topolinski S, Strack F. 2008. Where there's a will-there's no intuition. J Mem Lang 58: 1032-1048.

Topolinski S, Strack F. 2009. The analysis of intuition: processing fluency and affect in judgments of semantic coherence. Cogn Emot 23: 1465-1503.

Tucker MA, Fishbein W. 2008. Enhancement of declarative memory performance following a daytime nap is contingent on strength of initial task acquisition. Sleep 31: 197-203.

Verleger R, Rose M, Wagner U, Yordanova J, Kolev V. 2013. Insights into sleep's role for insight: Studies with the number reduction task. Adv Cogn Psychol 9: 160-172.

Volz KG, Zander T. 2014. Primed for intuition? Neurosci Dec Mak 1: 26-34.

Wilhelm I, Diekelmann S, Molzow I, Ayoub A, Mölle M, Born J. 2011. Sleep selectively enhances memory expected to be of future relevance. $J$ Neurosci 31: 1563-1569.

Wilhelm I, Metzkow-Mészàros M, Knapp S, Born J. 2012. Sleep-dependent consolidation of procedural motor memories in children and adults: the pre-sleep level of performance matters. Dev Sci 15: 506-515.

Yaniv I, Meyer DE. 1987. Activation and metacognition of inaccessible stored information: Potential bases for incubation effects in problem solving. J Exp Psychol Learn Mem Cogn 13: 187-205.

Yordanova J, Kolev V, Verleger R, Bataghva Z, Born J, Wagner U. 2008. Shifting from implicit to explicit knowledge: different roles of early and late night sleep. Learn Mem 15: 508-515.

Received October 28, 2016; accepted in revised form August 25, 2017. 


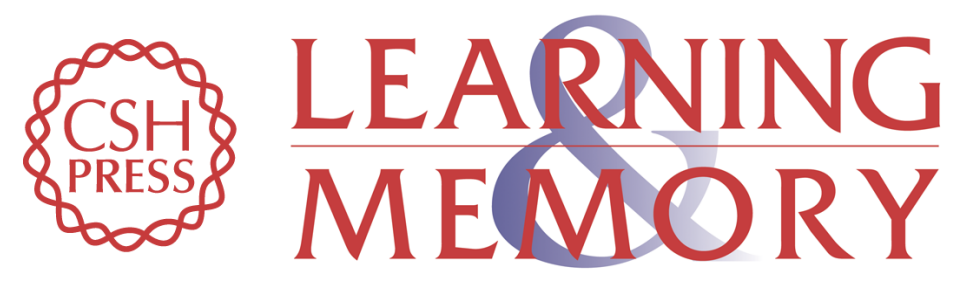

\section{Sleep increases explicit solutions and reduces intuitive judgments of semantic coherence}

Thea Zander, Kirsten G. Volz, Jan Born, et al.

Learn. Mem. 2017, 24:

Access the most recent version at doi:10.1101/Im.044511.116

\section{Supplemental http://learnmem.cshlp.org/content/suppl/2017/11/14/24.12.641.DC1 Material}

References This article cites 37 articles, 4 of which can be accessed free at: http://learnmem.cshlp.org/content/24/12/641.full.html\#ref-list-1

Creative This article is distributed exclusively by Cold Spring Harbor Laboratory Press for the Commons first 12 months after the full-issue publication date (see

License http://learnmem.cshlp.org/site/misc/terms.xhtml). After 12 months, it is available under a Creative Commons License (Attribution-NonCommercial 4.0 International), as described at http://creativecommons.org/licenses/by-nc/4.0/.

Email Alerting Receive free email alerts when new articles cite this article - sign up in the box at the Service top right corner of the article or click here. 